\title{
A case of the tail wagging the dog
}

The N-terminal tail of histone proteins provides a platform for post-translational modifications that are important for gene activity. One such modification - the acetylation of histone H4 at Lys16 (H4K16Ac) - is associated with transcriptional activation and the maintenance of euchromatin. Craig Peterson and colleagues now report in Science that this single histone modification inhibits chromatin folding, and thereby keeps the chromatin in an 'open' configuration that allows gene transcription to take place.

Previous studies have shown that a region of the $\mathrm{H} 4$ tail containing residue K16 is important for the

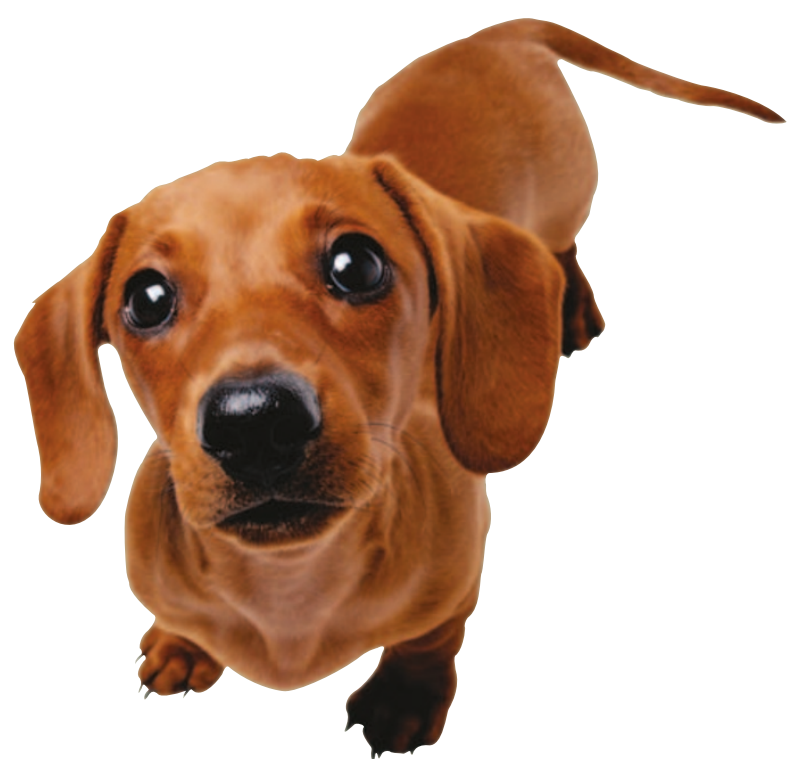

formation of 30-nm chromatin fibres. To test whether the modification of K16 might control higher-order chromatin formation, Peterson and colleagues chemically synthesized an H4-tail peptide that contained an acetylated K16 residue and attached it to the remaining part of the $\mathrm{H} 4$ protein. The recombinant $\mathrm{H} 4$ protein was incorporated into histone octamers, which were assembled with a DNA template into nucleosomal arrays.

Under conditions in which the nucleosomal arrays adopted a 'beads-on-a-string' conformation, arrays that contained either the wild-type histone $\mathrm{H} 4, \mathrm{H} 4 \mathrm{~K} 16 \mathrm{Ac}$ or $\mathrm{H} 4-\Delta \mathrm{N}$ (which lacks the $\mathrm{N}$-terminal tail region) were analysed, and all showed nearly identical sedimentation coefficients. However, by adding magnesium salt, which promotes chromatin compaction, the average sedimentation coefficient of the wild-type $\mathrm{H} 4$ arrays increased, which was consistent with the formation of compact, 30-nm-like fibres. By contrast, arrays that contained either $\mathrm{H} 4 \mathrm{~K} 16 \mathrm{Ac}$ or H4- $\Delta \mathrm{N}$ were unable to condense fully and showed only a moderate increase in the average sedimentation coefficient.

A further increase in the magnesium-salt concentration promotes the self-association of the arrays through fibre-fibre interactions that stabilize higher-order chromatin domains. Self-association of the arrays that contained H4K16Ac or H4- $\Delta \mathrm{N}$ required higher concentrations of magnesium salt compared with those comprising wild-type $\mathrm{H} 4$. So, the acetylation of H4K16 had the same effect as deleting the entire $\mathrm{H} 4$ tail and inhibits 30-nm fibre formation as well as cross-fibre interactions. Indeed, H4K16Ac was associated with decondensed chromatin in vivo, as nucleosomal arrays that contained H4K16Ac were present predominantly in $\mathrm{MgCl}_{2}$-soluble chromatin fractions, which are known to be enriched in transcriptionally active genes.

Finally, the Peterson team tested whether the H4K16Ac modification affects the function of the ATPdependent chromatin assembly and remodelling factor (ACF) complex, which associates with the $\mathrm{H} 4$ tail to enable nucleosomes to slide along the DNA. They showed that wildtype mononucleosomes that had been incubated with ACF exhibited significant sliding, whereas sliding by H4K16Ac-containing nucleosomes was reduced.

These results show that a single histone modification can dramatically change the state of chromatin by inhibiting the formation of higherorder chromatin and regulating the activity of a chromatin remodelling enzyme, thereby keeping the chromatin in a decondensed, transcriptionally active conformation.

Arianne Heinrichs

ORIGINAL RESEARCH PAPER Shogren-Knaak M. et al. Histone H4-K16 acetylation controls chromatin structure and protein interactions. Science 311, 844-847 (2006) 